\title{
Recombinant Adeno-Associated Virus Expressing Truncated IK Cytokine Diminishes the Symptoms of Inflammatory Arthritis ${ }^{\text {s }}$
}

\author{
Seulgi Choi ${ }^{1}$, Hyelim Park ${ }^{1}$, Marstella Minelko ${ }^{1}$, Eun-Kyung Kim ${ }^{2}$, Mi-Ra Cho ${ }^{2}$, and Jae-Hwan Nam ${ }^{1 *}$ \\ ${ }^{1}$ Department of Biotechnology, The Catholic University of Korea, Bucheon 14662, Republic of Korea \\ ${ }^{2}$ The Rheumatism Research Center, Catholic Research Institute of Medical Science, College of Medicine, The Catholic University of Korea, \\ Seoul 06591, Republic of Korea
}

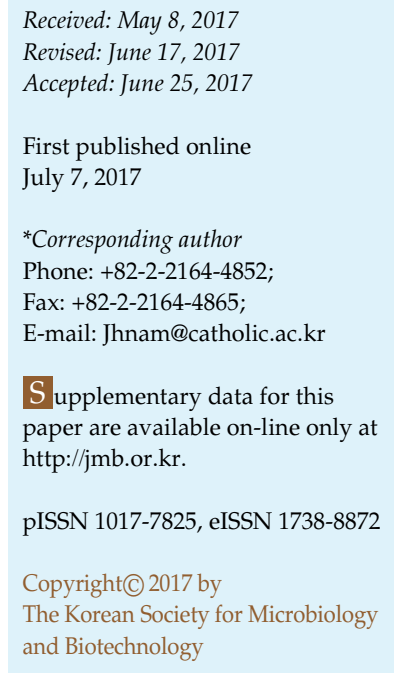

IK can downregulate interferon-gamma-induced major histocompatibility complex (MHC) class II expression through the MHC class II transactivator, which suggests that IK can inhibit the interactions between immune cells. We delivered adeno-associated virus serotype 2 (AAV2) encoding the genes for truncated IK (tIK) or green fluorescent protein (GFP) to DBA1/J mice via intravenous injection. Seven weeks after injection, collagen-induced arthritis was induced in the AAV2-treated mice. AAV2-tIK injection reduced the severity of arthritis and the percentage of pathogenic Th17 cells compared with AAV2-GFP injection. These results suggest a novel gene therapy strategy for treatment of inflammatory arthritis.

Keywords: Adeno-associated virus, gene therapy, inflammatory arthritis, rheumatoid arthritis, truncated IK
IK functions as a downregulator of the expression of major histocompatibility complex (MHC) class II that is induced by interferon (IFN)- $\gamma$. This function of IK is associated with the MHC class II transactivator, which is an influential player in both constitutive and cytokineinducible expression of MHC class II [1]. Many studies have clearly demonstrated that MHC class II is an important factor in antigen recognition. Moreover, excessive expression of MHC class II has been recognized as one cause of autoimmune diseases, such as rheumatoid arthritis (RA), psoriasis, and lupus nephritis [2]. The ability of IK to downregulate MHC class II expression suggests that it has the potential to be applied to therapy of autoimmune diseases. Indeed, previous studies have demonstrated the potential of IK as a therapeutic agent for autoimmune diseases, including lupus nephritis and RA. Treatment of MRL/lpr mice with truncated IK (tIK) reduced renal damage through reduction of macrophage and $\mathrm{T}$ cell infiltration [3]. Our previous study showed that transgenic mice expressing tIK were resistant to the induction of inflammatory arthritis via a reduction in proinflammatory immune cells such as macrophages and pathogenic T helper 1 (Th1) and Th17 cells [4]. These previous reports indicated that IIK may be a candidate for treatment of inflammatory arthritis, such as RA.

Adeno-associated virus (AAV) is recognized as a promising vehicle for gene delivery for gene therapy. AAV has several advantages compared with other viral vectors [5], including a good level of safety because of its lack of pathogenicity and immunogenicity. It also has other useful characteristics, such as the ability of long-term expression of inserted genes and tropism for a broad range of cells [6].

In this study, we examined the therapeutic effect of tIK for RA using recombinant AAV serotype 2 expressing tIK (AAV2-tIK), which was applied to collagen-induced arthritis (CIA) in DBA/1J mice as an experimental model of arthritis.

We cloned the tIK gene fused with the hemagglutinin (HA) gene into the AAV2 viral vector and pcDNA3.1. The tIK-HA gene was obtained from a previous clone [4]. The 
recombinant AAV2-tIK was constructed by Virovek (USA). The expression of the tIK gene in 293T cells by infection with $1 \times 10^{11}$ and $1 \times 10^{12}$ viral genomes $(\mathrm{vg}) / \mathrm{ml}$ of recombinant AAV2-tIK and transfection with pcDNA3.1tIK (tIK-HA) as the positive control were confirmed. Recombinant AAV2-GFP as a negative control was purchased from Virovek. Expression of tIK was confirmed using anti-HA antibody (Bethyl Laboratories, USA) and anti-RED antibody (Thermo Fisher Scientific, USA) to detect tIK and the fused protein. RED is a unique aminoacid sequence characteristic of IK [7]. The expression of tIK in $293 \mathrm{~T}$ cells in vitro was confirmed to be proportional to the amount of AAV2-tIK vector, and the size of the tIK expressed after AAV2-tIK infection was exactly the same as that of the transfected tIK-HA fusion protein detected by both anti-HA and anti-RED antibodies (Fig. 1A). To confirm the tIK expression in mice in vivo, the mRNA expression level of tIK was analyzed in the liver and spleen of injected mice at 6 weeks after injection. In liver tissue, the tIK mRNA expression level was detected in AAV2-tIKinjected mice, but not in AAV2-GFP-injected mice. The tIK mRNA expression level in spleen tissue was not detected in both AAV2-tIK- and AAV2-GFP-injected mice (Fig. 1B). However, the results were not statistically significant.

Next, we injected $2 \times 10^{11} \mathrm{vg} /$ mouse of AAV2-tIK or AAV2-GFP intravenously into DBA/1J mice twice at 1week intervals. At 7 weeks after AAV2-tIK or AAV2-GFP injection, we induced CIA in the DBA/1J mice as an experimental model of arthritis. An emulsion of $10 \mathrm{mg} / \mathrm{ml}$ collagen and complete Freund's adjuvant (first injection) or incomplete Freund's adjuvant (booster injection 2 weeks later) was injected intradermally [8]. All animal studies were approved by and performed according to the Institutional Animal Care and Use Committee of the Sungsim Campus at the Catholic University of Korea (IACUC Board Regulations \#2016-009) [9]. To analyze the RA symptoms, the severity of symptoms in each paw was scored on a scale from 0 (no symptoms) to 4 (maximal level of symptoms), and the scores for the four limbs were summed for each mouse $(n=6)$. The severity and incidence of arthritis were scored at intervals of 3-5 days over 4 weeks. The severity and incidence of RA were lower in the AAV2-tIK-injected mice than in the mice treated with AAV2-GFP during the observation days (28 days after collagen injection) (Figs. 2A and 2B). Mice were sacrificed for analysis 28 days after the booster immunization. We observed less destruction in the joints of the AAV2-tIKinjected mice compared with that of the AAV2-GFPinjected mice (Fig. 2C). Histological analysis showed that the levels of inflammatory cytokines (IL-1 $\beta$, TNF- $\alpha$, and IL17) and pathogenic factors related to RA (RANKL and MMP9) were lower in the joints of AAV2-tIK-injected mice than in those of AAV2-GFP-injected mice (Fig. 2D). The percentages of Th1 and Th17 cells in splenocytes were analyzed using anti-CD4-allophycocyanin as a surface marker, and anti-IFN- $\gamma$-phycoerythrin (PE) and anti-IL17A-PE as an intracellular marker. The pseudocolor dot plots represent cell distribution gated on $\mathrm{CD} 4^{+} \mathrm{T}$ cells. Flow cytometric analysis of splenocytes showed that the proportions of Th1 and Th17 cells were lower in AAV2-tIKinjected mice than in AAV2-GFP-injected mice (Fig. 2E). A previous report showed that tIK may only function in an
A

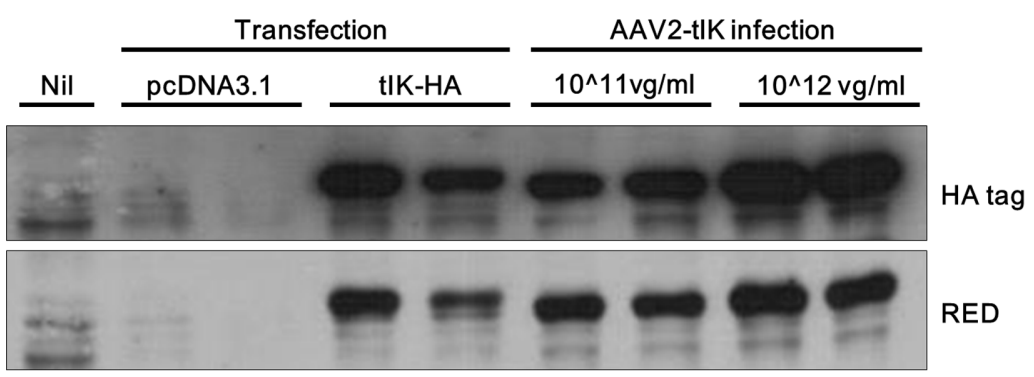

B

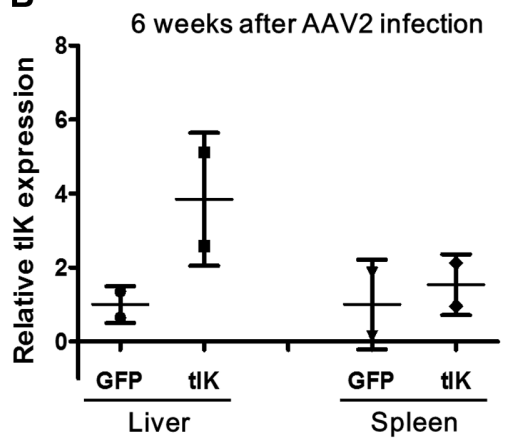

Fig. 1. Expression of tIK gene by recombinant AAV2-tIK.

(A) tIK gene expression by tIK-pcDNA3.1 plasmid (tIK-HA) and recombinant AAV2-tIK in vitro were compared by western blot analysis. Nil: Nontreated 293T cells; pcDNA3.1: 293T cells transfected with empty pcDNA3.1 vector; tIK-HA: 293T cells transfected with tIK-HA in pcDNA3.1 plasmid; $1 \times 10^{11}$ and $1 \times 10^{12}$ viral genomes $(\mathrm{vg}) / \mathrm{ml}$ : recombinant AAV2-tIK-infected 293T cells. (B) The expression of the tIK gene by recombinant AAV2-tIK in vivo was detected by quantitative PCR. Six weeks after injection of $2 \times 10^{11} \mathrm{vg} / \mathrm{mouse}$ of AAV2-GFP or AAV2-tIK, the tIK gene expression level was quantitated and compared between the two groups. 
A
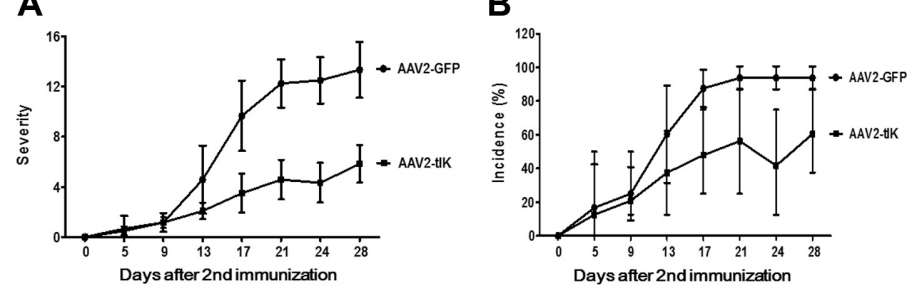

C

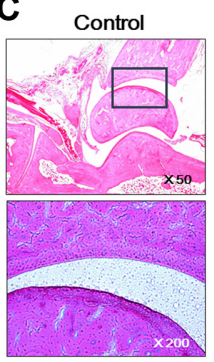

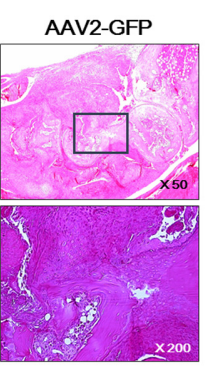

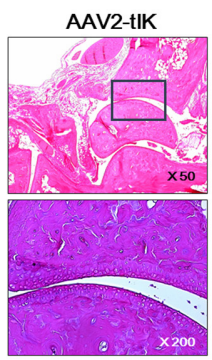

D

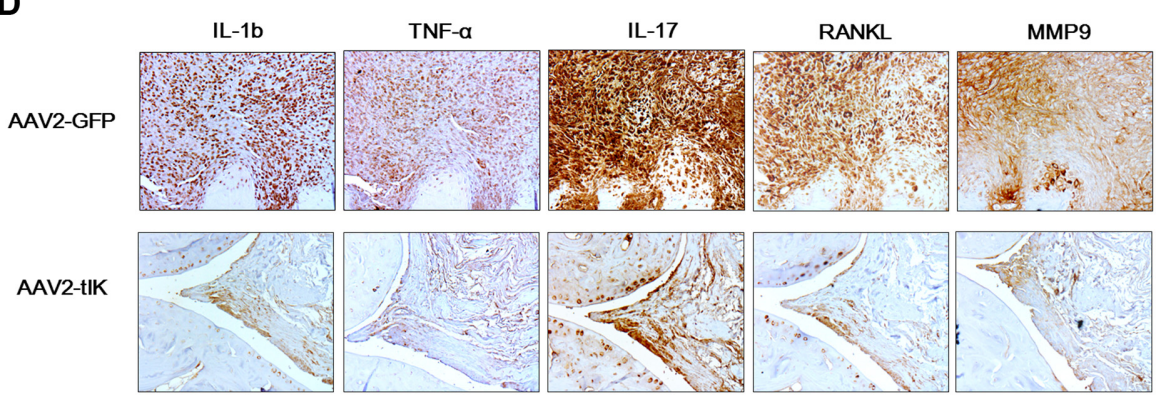

E
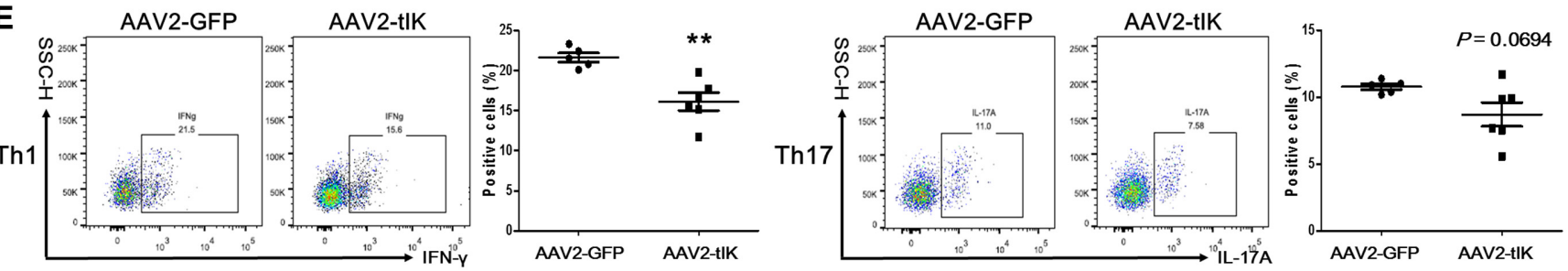

Fig. 2. Effect of AAV2-tIK in the CIA mouse model.

(A) The severity and (B) incidence of arthritis in the collagen-induced arthritis (CIA) mouse model were compared. (C) The mouse ankle-joint tissue sections were stained with hematoxylin and eosin. (D) Inflammatory cytokines and molecules related to rheumatoid arthritis were analyzed by immunohistochemistry staining. A representative image for $(\mathbf{C})$ and $(\mathbf{D})$ was selected for each group $(n=6)$. (E) Flow cytometric analysis of Th1 and Th17 cells in the CIA mouse model. The stacked bar graph shows the mean percentages. Data are represented as the mean $\pm \operatorname{SD}(n=5-6)$. ${ }^{* *} p$ $<0.005$. Control: a normal mouse joint; AAV2-GFP: the joint of a CIA mouse injected with $2 \times 10^{11}$ viral genomes (vg)/mouse of recombinant AAV2-GFP; AAV2-tIK: the joint of a CIA mouse injected with $2 \times 10^{11} \mathrm{vg} /$ mouse of recombinant AAV2-tIK.

immunologically activated condition, but not in a normal condition [4]. Therefore, AAV2-tIK may not show any immunological response in a normal condition in the mouse.

RA is a chronic inflammatory disease characterized by synovial inflammation and joint damage. Although the causes of RA are unclear, it is considered that not only the individual's genetic and environmental background but also the infiltration of immune cells can influence the pathology of RA [10]. IL-1 $\beta$ and TNF- $\alpha$ are typical proinflammatory cytokines associated with the disease [11-13], whereas IL-17 is mostly produced by Th17 cells and is related to the induction and pathogenesis of inflammatory arthritis [14]. Because of the relationships between these cytokines and RA, monoclonal antibodies targeting the cytokines have been used as therapy for inflammatory autoimmune diseases, including RA [15]. In addition, the RANKL-RANK receptor pathway stimulates the activation and differentiation of mature osteoclasts, inducing bone destruction in RA [16]. Because the MMP-9 level is high in serum and synovial fluids of RA patients, it has been highlighted as a significant marker of RA [17]. Our study showed that infection with AAV2-tIK reduced the levels of these proinflammatory cytokines in joint tissue (Fig. 2D) and reduced the symptoms of inflammatory arthritis (Figs. 2A and 2B), the destruction of joint tissue (Fig. 2C), and the proportions of proinflammatory cells such as Th1 and Th17 (Fig. 2E). All data clearly show that recombinant AAV2-tIK prevented the induction of inflammatory arthritis symptoms in the CIA disease 
model.

Although our previous study showed that tIK is a candidate target for inflammatory arthritis treatment [4], we did not then have a method for delivery of exogenous tIK into patients. In this study, we developed the recombinant AAV2-tIK for this purpose and showed that it has potential as a therapeutic agent for suppressing the progression of inflammatory arthritis. Moreover, it may be useful for gene therapy for RA (a type of inflammatory arthritis) patients because it is a viral vector system that can deliver therapeutic genes to these patients without repeated treatments.

\section{Acknowledgments}

The detailed protocols for all experiments are included in the supplementary data. This work was supported by the Catholic University of Korea, Research Fund 2017, the Ministry of Health \& Welfare, Republic of Korea (HI15C2955 and HI13C0826), KFDA (16172INFECTION268), and Basic Science Research Program through the NRF funded by the Ministry of Science, ICT \& Future Planning (NRF2015M3A9B5030157).

\section{References}

1. Vedrenne J, Assier E, Pereno R, Bouzinba-Segard $H$, Azzarone B, Jasmin C, et al. 1997. Inhibitor (IK) of IFN-g induced HLA class II antigens expression also inhibits HLA class II constitutive expression in the human Raji B cell line. Oncogene 14: 1453-1461.

2. Tsai S, Santamaria P. 2013. MHC class II polymorphisms, autoreactive T-cells, and autoimmunity. Front. Immunol. 4: e000321.

3. Muraoka M, Hasegawa H, Kohno M, Inoue A, Miyazzaki T, Terada $\mathrm{M}$, et al. 2006. IK cytokine ameliorates the progression of lupus nephritis in MRL/lpr mice. Arthritis Rheumatol. 54: 3591-3600.

4. Park HL, Lee SM, Min JK, Moon SJ, Kim I, Kang KW, et al. 2017. IK acts an immunoregulator of inflammatory arthritis by suppressing Th17 cell differentiation and macrophage activation. Sci. Rep. 7: 40280.

5. Daya S, Berns KI. 2008. Gene therapy using adenoassociated virus vectors. Clin. Microbiol. Rev. 16: 1073-1080.

6. Kotterman MA, Schaffer DV. 2014. Engineering adenoassociated viruses for clinical gene therapy. Nat. Rev. Gene 15: 445-451.

7. Assier E, Bouzinba-Segard H, Stolzenberg MC, Stephens R, Bardos J, Freemont P, et al. 1999. Isolation, sequencing and expression of RED, a novel human gene encoding an acidicbasic dipeptide repeat. Gene 230: 145-154.

8. Brand DD, Latham KA, Rosloniec EF. 2007. Collageninduced arthritis. Nat. Protoc. 2: 1269-1275.

9. Cho A, Seok SH. 2013. Ethical guidelines for use of experimental animals in biomedical research. J. Bacteriol. Virol. 43: 18-26.

10. Mellado M, Martínez-Muñoz L, Cascio G, Lucas P, Pablos JL, Rodríguez-Frade JM. 2015. T cell migration in rheumatoid arthritis. Front. Immunol. 6: 384.

11. Hernandez-Pando R, Rook GA. 1997. The role of TNF-a in T-cell-mediated inflammation depends on the Th1/Th2 cytokine balance. Immunology 82: 591-595.

12. Lichtman AH, Chin J, Schmidt JA, Abbas AK. 1988. Role of interleukin 1 in the activation of T lymphocytes. Proc. Natl. Acad. Sci. USA 85: 9699-9703.

13. Wakil AE, Wang ZE, Ryan JC, Fowell DJ, Locksley RM. 1998. Interferon gamma derived from CD4(+) $\mathrm{T}$ cells is sufficient to mediate $\mathrm{T}$ helper cell type 1 development. $J$. Exp. Med. 188: 1651-1656.

14. Paradowska-Gorycka A, Haladyj E. 2015. Th17-cells in the pathogenesis of rheumatoid arthritis. Int. J. Autoimmune Disord. Ther. 1: 101.

15. Tanaka T, Hishitani Y, Ogata A. 2014. Monoclonal antibodies in rheumatoid arthritis: comparative effectiveness of tocilizumab with tumor necrosis factor inhibitors. Biologics 8: 141-153.

16. Tanaka S. 2013. Regulation of bone destruction in rheumatoid arthritis through RANKL-RANK pathways. World J. Orthop. 4: $1-6$.

17. Mohammed FF, Smookler DS, Khokha R. 2003. Metalloproteinases, inflammation, and rheumatoid arthritis. Ann. Rheum. Dis. 62 (Suppl. 2): ii43-ii47. 